\title{
An experimental rabbit model of symptomatic cerebral vasospasm with in vivo neuroimaging assessment and ex vivo histological validation
}

\author{
YUNCHANG MO $^{1 *}$, LUPING HUANG $^{1 *}$, LINBI CHEN $^{1 *}$, MICHAEL VERONESI $^{2}$, YIYI SHI $^{1}$, \\ SIJIA CHEN ${ }^{1}$, LINLI PENG ${ }^{1,3}$, LEPING ZHOU ${ }^{1,4}$, YONGLIN PU ${ }^{2}$ and JUNLU WANG ${ }^{1}$ \\ ${ }^{1}$ Department of Anesthesia, The First Affiliated Hospital of Wenzhou Medical University, Wenzhou, \\ Zhejiang 325000, P.R. China; ${ }^{2}$ Department of Radiology, University of Chicago Hospital, Chicago, IL 60637, USA; \\ ${ }^{3}$ Department of Anesthesia, Guangdong Provincial Maternity and Child Care Center, Guangzhou, Guangdong 510010; \\ ${ }^{4}$ Department of Anesthesia, Zhejiang Provincial People's Hospital, Hangzhou, Zhejiang 310014, P.R. China
}

Received August 11, 2016; Accepted July 27, 2017

DOI: $10.3892 /$ etm.2018.5690

\begin{abstract}
Cerebral vasospasm (CVS) is a severe complication that occurs following aneurysmal subarachnoid hemorrhage (SAH). Magnetic resonance angiography (MRA) has been used to evaluate brain injury following SAH in humans. The present study was designed to assess a rabbit model of symptomatic CVS (SCVS) and the utility of MRA in evaluating SCVS in rabbits. Japanese white rabbits $(n=24)$ were randomly divided into 2 equal groups: A sham group and a SAH group. Neurological scores were evaluated for 7 days following SAH. Basilar artery (BA) diameters were measured using MRA preoperatively and 7 days postoperatively. Rabbits were sacrificed 7 days following SAH and the BA diameter of each rabbit was determined using histological evaluation. Compared with the Sham group, neurological function was significantly reduced in the SAH group at all time points $(\mathrm{P}<0.05)$. Furthermore, the BA diameter was significantly smaller in the SAH group on day 7 compared with the baseline measurement $(\mathrm{P}<0.05)$. No significant difference was observed between histological and MRA findings in either group at day 7. Histological changes in the hippocampus consistent with ischemia were observed in the SAH group. Hippocampal ischemia was also identified in
\end{abstract}

Correspondence to: Professor Yonglin $\mathrm{Pu}$, Department of Radiology, University of Chicago Hospital, 5841 South Maryland Avenue, Chicago, IL 60637, USA

E-mail: ypu@radiology.bsd.uchicago.edu

Professor Junlu Wang, Department of Anesthesia, The First Affiliated Hospital of Wenzhou Medical University, 2 Fuxue Road, Wenzhou, Zhejiang 325000, P.R. China

E-mail: wangjunlu973@163.com

${ }^{*}$ Contributed equally

Key words: magnetic resonance angiography, subarachnoid hemorrhage, cerebral vasospasm, animal model the SAH group via MRA and there was no difference in detection rates following the use of MRA and histochemistry. MRA appears to be an effective method for assessing vasospasms of the BA and ischemic changes to the hippocampus in a rabbit model of SCVS. Furthermore, the animal model used in the present study may be beneficial for the future study of SCVS.

\section{Introduction}

Patients with subarachnoid hemorrhage (SAH) often experience life-threatening complications (1). Aneurysmal SAH (aSAH) is a neurological condition with high mortality $(>25 \%)$ and significant morbidity ( $>50 \%$ ) rates amongst survivors (2).

Cerebral vasospasm (CVS) is the leading cause of morbidity and mortality following aSAH (3). Vasospasm occurs in $50-70 \%$ of patients with $\mathrm{SAH}$ and $50 \%$ of these patients experience neurological symptoms in a condition known as symptomatic cerebral vasospasm (SCVS) (1). Cerebral infarction occurs in half of all patients with SCVS and is fatal in $30 \%$ of patients (4). It is important to study CVS vasospasm in order to develop effective treatments and reduce the morbidity rate of patients with this condition.

Current treatments for SCVS following SAH include, triple-H therapy, prophylactic hyperdynamic postoperative fluid therapy and drug therapy (5-7). However, they are limited as the mechanism of action underlying the condition remains poorly understood. Establishing an effective model of SCVS has therefore been a focus of neurological research since 1961 (8). Several different animal models have been developed, including rat, rabbit, dogs, monkey and pig models (9-13). There are $\sim 50$ different animal models of CVS; however, the majority have limited utility as animals are typically asymptomatic and only a few, including rabbits and monkeys, exhibit symptomatic neurological deficits (14). The monkey model is expensive and not readily available (14), therefore it is important to establish a reliable symptomatic SCVS model using rabbits.

Researchers have measured the diameter of the basilar artery (BA) using computed tomographic angiography (CTA) to evaluate CVS (15). CTA is an effective method of assessing 
the occurrence and extent of BA spasms; however, it requires repeated venopuncture of the ear vein and high doses of contrast agents, which may damage the ear vein and adversely affect renal function $(16,17)$. Bilateral carotid artery ligation is required to induce SCVS in the rabbit model and in studies conducting CTA, the decision to include a particular rabbit is based on its neurological score following bilateral carotid artery ligation (18). This evaluation tends to be subjective and may affect the result of the experiment (18).

Delayed infarction is the most important modifiable factor that affects quality of life following SAH (19). CTA is not useful for evaluating infarctions in brain tissue, whereas magnetic resonance angiography/magnetic resonance imaging (MRA/MRI) are highly sensitive and specific (20). MRA is widely used in clinical settings as no contrast agent is required to measure the BA diameter and the ischemic area may easily be observed $(21,22)$. However, to the best of our knowledge, the use of MRA in a rabbit SCVS model has not been previously reported.

The aim of the present study was to evaluate the feasibility of using MRA to assess a modified rabbit SCVS model by measuring the BA diameter and ischemic area following CVS. These measurements were then compared with those obtained by direct pathological examination.

\section{Materials and methods}

Ethical approval. The protocol followed in the current study was approved by the Special Committee on Animal Welfare of Wenzhou Medical University (Wenzhou, China). All animals were treated humanely in accordance with the guidelines for the Care and Use of Laboratory Animals published by the U.S. National Institutions of Health (NIH Publication No. 85-23, revised 1996).

CVS model. A total of 24 male Japanese white rabbits (2-3 months; weight $2.5-3.0 \mathrm{~kg}$ ) were purchased from the Wenzhou Experimental Animal Center (Wenzhou, China). The animals had free access to standard chow and tap water in a temperature-controlled chamber at $24^{\circ} \mathrm{C}$ with a $12 \mathrm{~h}$ light/dark cycle. Rabbits were randomly assigned to one of two groups ( $n=12$ each): A sham group and a SAH model group. The sham group received a $1.0 \mathrm{ml} / \mathrm{kg}$ saline injection into the cistern and the SAH model group received a $1.0 \mathrm{ml} / \mathrm{kg}$ autologous blood injection into the subarachnoid spaces. These injections were perfomed twice, with an interval of $48 \mathrm{~h}$ between them.

CVS was induced following $\mathrm{SAH}$, as previously reported (15). In brief, rabbits were anesthetized with intramuscularly injected ketamine $(25 \mathrm{mg} / \mathrm{kg}$; cat. no. 1507294; Fujian Gutian Pharmaceutical Co. Ltd., Ningde, China) and promethazine (12.5 mg/kg; cat. no. 13160301; Shanghai Hefeng Pharmaceutical Co. Ltd., Shanghai, China), and bilateral carotid artery ligation was subsequently performed. Following 2 weeks, the rabbits were evaluated using MRA/MRI to measure the basilar artery (BA) diameter and evaluate whether brain infarction had occurred. If brain infarction, severe neurological symptoms or mortality were observed in any of the rabbits at 2 weeks they were replaced to ensure that each group contained 12 rabbits (Table I).

Following MRA, rabbits in the SAH group were extended in a lateral position during spontaneous breathing. The atlanto-occipital membrane was pierced with a 25 -gauge needle inserted into the cisterna magna, an attached syringe was subsequently used to remove the cerebrospinal fluid. The needle pierced the atlanto-occipital membrane and $1.0 \mathrm{ml} / \mathrm{kg}$ cerebrospinal fluid was extracted. An equal volume of fresh non-heparinized autologous arterial blood was obtained from the ear artery following the extraction of the cerebrospinal fluid. This was injected into the cisterna magna within 2 min. Arterial blood was analyzed using an ABL90 FLEX blood gas analyzer (Radiometer Medical ApS, Copenhagen, Denmark), which measured the $\mathrm{PO}_{2}$ and $\mathrm{PCO}_{2}$. The cisterna magna was re-punctured $48 \mathrm{~h}$ later and autologous arterial blood injection was repeated.

Neurological testing was performed every day following the establishment of CVS and SAH, as previously reported (23). Neurological deficits were graded using a four-point system by observing the rabbits on a flat surface, with lower scores indicating better neurological function. Rabbits were assessed by two blinded independent investigators.

MRA/MRI evaluation. BA diameters were measured using an SIGNA HDx MRI 3.0 machine (GE Healthcare Bio-Sciences, Pittsburgh, PA, USA) 1 day prior to the injection of blood (day 0 ) and 7 days following SAH. The rabbits were anesthetized with intramuscularly injected ketamine $(25 \mathrm{mg} / \mathrm{kg})$ and promethazine $(12.5 \mathrm{mg} / \mathrm{kg})$, and maintained in the left lateral position. The T2 sequence of brain scans used the following acquisition parameters: Repetition time (TR) 2,500 msec; echo time (TE) $96.9 \mathrm{msec}$; field of view (FOV), 7x7 cm . Three-dimensional time-of-flight (3D-TOF) MRA was used with the following acquisition parameters: TR, $31 \mathrm{msec}$; TE, $7.7 \mathrm{msec}$; FOV, 10x10 $\mathrm{cm}^{2}$. Images were transferred to a workstation and processed using the image-processing software (both GE Healthcare Bio-Sciences). The BA diameter of each rabbit was measured by two experienced radiologists. The BA diameter was measured in three individual segments: Proximal, middle and distal (24). Images were evaluated for abnormal signals indicating brain lesions in the hippocampal region and the number of abnormal signals was recorded.

Histological evaluation. The rabbits were sacrificed at 7 days following SAH and the BA and hippocampus were subsequently harvested by perfusion-fixation. The thorax was opened and a cannula was introduced into the left ventricle. The descending thoracic aorta was clamped and the right atrium was opened. Perfusion was initiated with $500 \mathrm{ml}$ physiological PBS (pH 7.4) at $37^{\circ} \mathrm{C}$ for $10 \mathrm{~min}$, followed by $500 \mathrm{ml} 10 \%$ buffered formaldehyde at $37^{\circ} \mathrm{C}$ under a perfusion pressure of $120 \mathrm{~cm}$ water for $10 \mathrm{~min}$. The hippocampus and BA were fixed in $10 \%$ buffered formaldehyde for $24 \mathrm{~h}$ at room temperature, embedded in paraffin and sliced into $4-\mu \mathrm{m}$ sections with a microtome. The formalin-fixed, paraffin-embedded BA and hippocampus sections were subsequently deparaffinized, hydrated, washed and stained with hematoxylin and eosin (H\&E) for $1 \mathrm{~min}$ at room temperature. Micrographs of the BAs were observed through a light microscope (Olympus Corporation, Tokyo, Japan) and scanned into the computer (magnification, x400). The cross-sectional areas of blood vessels were measured using a high-definition medical image analysis program (HMIAP-2000, Tongji Medical University, Hubei, China). 
Table I. Rabbits excluded from the present study.

\begin{tabular}{lcccc}
\hline Group & Mortalities & Severe neurological symptoms & Brain infarction identified by MRI & Total excluded rabbits \\
\hline $\begin{array}{l}\text { Sham } \\
\text { Subarachnoid }\end{array}$ & 2 & 2 & 2 & 6 \\
hemorrhage & 2 & 2 & 3 & 7 \\
\hline
\end{tabular}

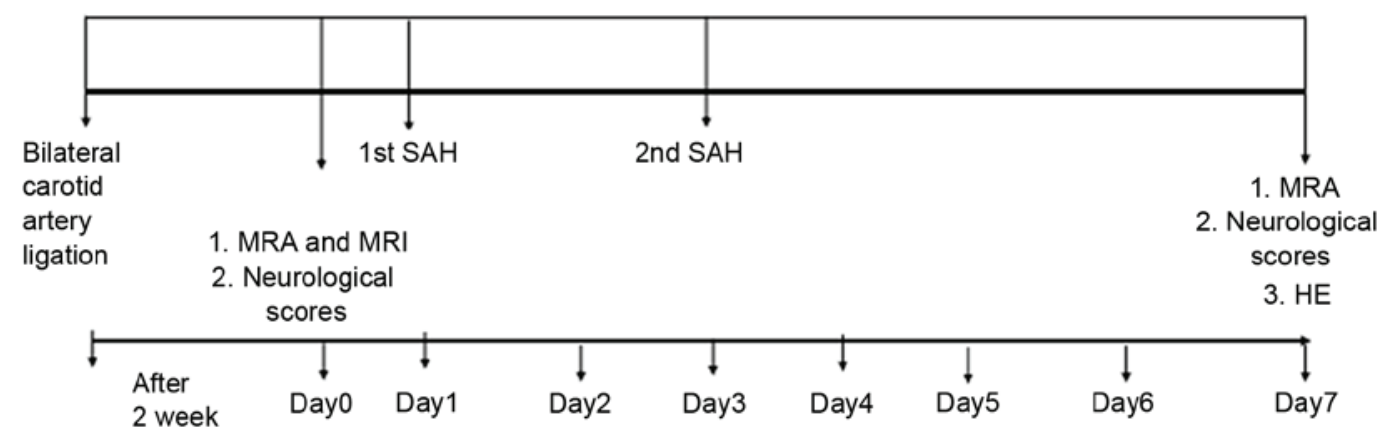

Figure 1. Diagram of the experimental protocol. SAH, subarachnoid hemorrhage; MRA, magnetic resonance angiography; MRI, magnetic resonance imaging; H\&E, hematoxylin and eosin staining.

For each vessel, three sequential sections (the midpoint of the proximal, middle and distal BA) were measured and the mean was calculated Each hippocampus was evaluated by blinded pathologists to identify the presence of karyopyknosis, cytoplasmic staining and smaller cell bodies in the hippocampal CA1 zone and the incidence of ischemia in all rabbits was recorded. The experimental protocol is presented in Fig. 1.

Statistical analysis. Statistical analyses were performed using SPSS (version 13.0; SPSS, Inc., Chicago, IL, USA). Differences in neurological scores between the two groups were assessed using the Wilcoxon rank sum test. A Student's t test was used to compare BA diameters at day 7, the arterial blood gas analyses and differences in neurological scores between the two groups. The BA diameters in the model group were compared at each time point using the paired t test. Pearson correlation was used to compare the methods of evaluation. The incidence of brain damage as measured by MRA and H\&E staining was analyzed using the $\chi^{2}$ square test. $\mathrm{P}<0.05$ was considered to indicate a statistically significant difference.

\section{Results}

CVS model. A total of 13 rabbits that underwent carotid ligation surgery were excluded from the present study as they succumbed, or developed severe neurological symptoms or brain infarction following carotid ligation, presumably due to the lack of collateral blood flow (Table I). The baseline physiological parameters of the two groups are summarized in Table II and no significant differences were observed between the groups at baseline. The mean neurological scores for the groups are presented in Fig. 2. Neurological impairment scores were significantly higher in the SAH group compared with the sham group at all time points post-surgery ( $\mathrm{P}<0.05$; Fig. 2$)$. Representative MRA images of BA diameters are presented in Fig. 3. No evident differences in BA diameter in the Sham group were observed between days 0 and 7 (Fig. 3A and B). However, in the SAH group, the BA diameter was markedly decreased at day 7 compared with day 0 (Fig. 3C and D). Quantitative analysis of MRA results determined that the BA diameter was significantly decreased at day 7 compared with day 0 in the SAH group $(1.48 \pm 0.08$ and $1.26 \pm 0.09 \mathrm{~mm}$, respectively; $\mathrm{P}<0.05$; Fig. 4 ).

Histological evaluation. Representative histological images of the BA from the Sham and SAH groups are presented in Fig. 5. The BA diameter on day 7 as measured using H\&E was significantly lower in the SAH group compared with the Sham group $(1.17 \pm 0.06$ and $1.49 \pm 0.09 \mathrm{~mm}$, respectively; $\mathrm{P}<0.05$; Fig. 4). The measurements obtained using MRA and $\mathrm{H} \& \mathrm{E}$ staining were compared and a significant positive correlation was detected between the results of $\mathrm{H} \& \mathrm{E}$ and MRA in the sham group $(\mathrm{r}=0.76, \mathrm{P}=0.004$; Fig. $6 \mathrm{~A})$ and $\mathrm{SAH}$ group $(\mathrm{r}=0.70, \mathrm{P}=0.011$; Fig. $6 \mathrm{~B})$ on day 7 .

$M R A / M R I$. Brain MRA did not reveal ischemic changes in either group following bilateral carotid artery ligation at 2 weeks (Fig. 7). No significant differences were observed prior to and following saline injection in the Sham group (Fig. 7A and B); however, large flake and patchy high density of the hippocampus was observed in the SAH group 7 days post injection (Fig. 7D) compared with pre-injection (Fig. 7C). Hippocampal H\&E staining revealed normal cells and cellular arrangements in the Sham group at day 7 (Fig. 8A-C). In the SAH group, H\&E staining revealed karyopyknosis, cytoplasmic staining and smaller cell bodies in the hippocampal CA1 zone 7 days post-injection, indicating the presence of hippocampal ischemia (Fig. 8D-F). No significant difference was observed in the incidence of hippocampal ischemia identified by MRA and H\&E staining in the SAH group (Table III). 
Table II. Summary of physiological parameters of the groups at baseline.

\begin{tabular}{lcccc}
\hline Group & $\mathrm{N}$ & $\mathrm{pH}$ & $\begin{array}{c}\mathrm{PO}_{2} \\
(\mathrm{~mm} \mathrm{Hg})\end{array}$ & $\begin{array}{c}\mathrm{PCO}_{2} \\
(\mathrm{~mm} \mathrm{Hg})\end{array}$ \\
\hline $\begin{array}{l}\text { Reference } \\
\text { range }\end{array}$ & & $7.28-7.52$ & $55-91$ & $24-39$ \\
$\begin{array}{l}\text { Sham } \\
\begin{array}{l}\text { Subarachnoid } \\
\text { hemorrhage }\end{array}\end{array}$ & 12 & $7.36 \pm 0.06$ & $90.4 \pm 11.65$ & $34.05 \pm 6.26$ \\
\hline
\end{tabular}

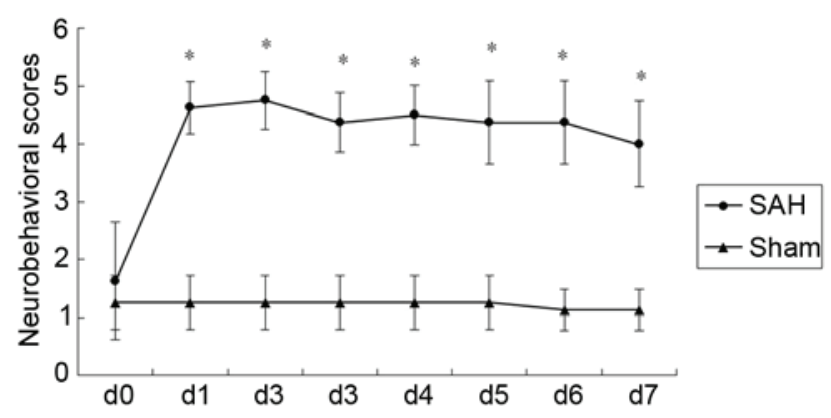

Figure 2. Neurobehavioral scores. The mean scores of the SAH group were significantly higher compared with the sham group on $\mathrm{d} 1-7$. ${ }^{*} \mathrm{P}<0.05$ vs. sham. SAH, subarachnoid hemorrhage; d, days.
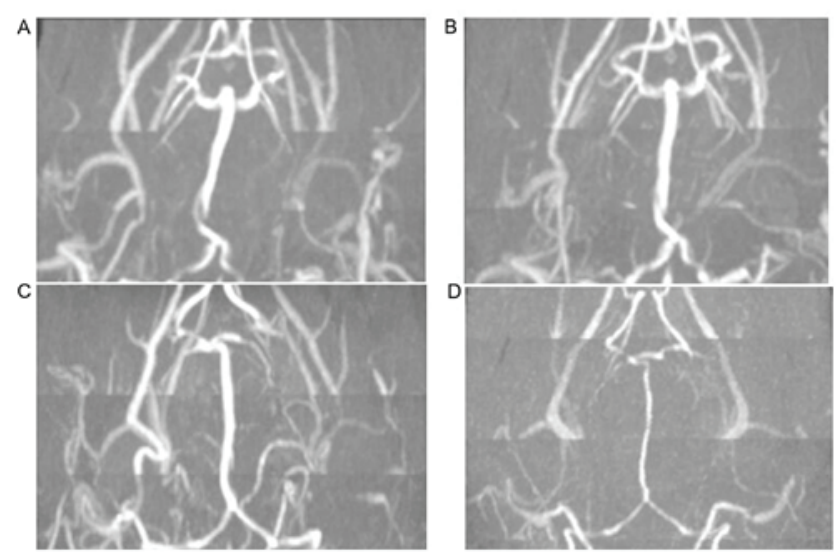

Figure 3. MRA images of BAs. MRA of the BA in the Sham group on days (A) 0 and (B) 7 and in the subarachnoid hemorrhage group on days (C) 0 and (D) 7. BA, basilar artery; MRA, magnetic resonance angiography. Magnification, $\mathrm{x} 1$.

\section{Discussion}

In the present study, a model of CVS in rabbits was successfully established and it was demonstrated that MRA may be used to accurately evaluate the degree of CVS. There was a strong correlation between H\&E staining of the BA and MRA for measuring the degree of vascular spasm. MRA revealed ischemia of the hippocampus 7 days following SAH and postoperative pathological examination indicated ischemia, indicating that MRA accurately identifies ischemia in the hippocampus.

$\mathrm{SAH}$ is a common acute and severe cerebral vascular disease and has the third highest incidence of all cerebrovascular

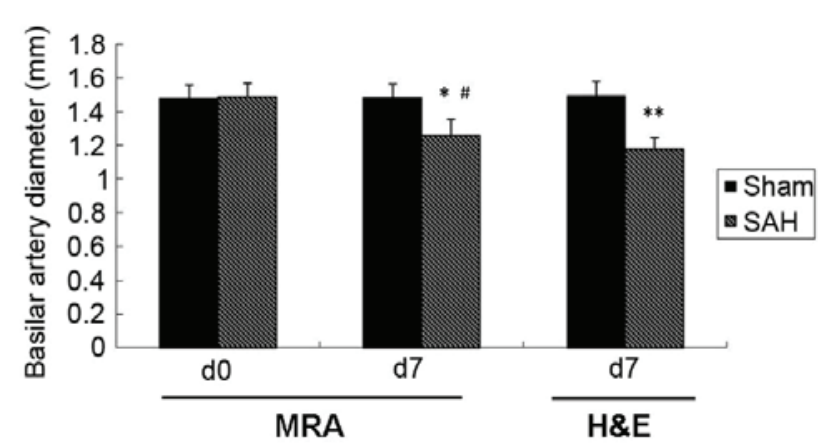

Figure 4. Changes in basilar artery diameter as measured using MRA and H\&E staining. ${ }^{*} \mathrm{P}<0.05$ vs. sham group at d7 by $\mathrm{MRA} ;{ }^{* *} \mathrm{P}<0.05$ vs. sham group at $\mathrm{d} 7$ by $\mathrm{H} \& \mathrm{E}$; ${ }^{\mathrm{P}}<0.05$ vs. $\mathrm{d} 0$ in $\mathrm{SAH}$ group by MRA. MRA, magnetic resonance angiography; $\mathrm{SAH}$, subarachnoid hemorrhage; H\&E, hematoxylin and eosin; d, days.
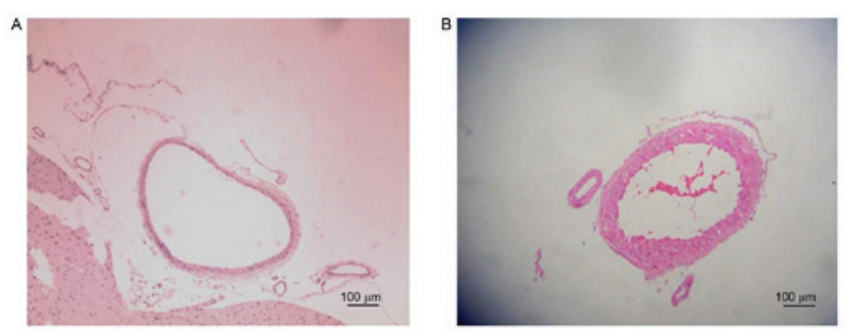

Figure 5. Basilar artery diameter assessed using hematoxylin and eosin staining. Representative images from the (A) Sham and (B) subarachnoid hemorrhage groups on day 7 .

diseases (25). SAH comprises only $5 \%$ of all strokes; however its mortality rate is high (40\%) due to delayed cerebral ischemia (DCI) and neurological deterioration occurring days following the hemorrhage (26). The severity of cerebral infarction and ischemia that occurs is associated with the severity of vasospasm observed on angiography (8). Delayed CVS may develop in patients with aneurysmal SAH (8). The severity and duration of vasospasm is associated with the thickness, density and persistence of the blood in the ventricle $(1,4)$. Delayed CVS with neurological dysfunction due to DCI is the primary cause of mortality following SAH (27). Furthermore, the primary cause of poor prognosis in patients with SAH is the insufficient treatment of CVS (28). To better understand the mechanism of delayed CVS with DCI and help identify a suitable therapy, it is necessary to establish a reliable animal model of symptomatic delayed CVS.

The majority of animal models of CVS establish only CVS without cerebral ischemia following SAH. Although SCVS may be simulated in primates, their utility in research is limited due to the high costs associated with keeping these animals. In rabbits, cerebral angiography may be repeated and the procedure for establishing a model of SAH with SCVS is relatively simple and inexpensive (12). Therefore, establishing a rabbit model of this condition is helpful to researchers. Using this model, the degree of CVS and cerebral ischemia may be determined by MRA/MRI. The rabbit model of CVS is considered to be superior to that of other animals (12). Compared with dogs or rats, the cerebral vascular system of rabbits is more similar to the human system, as it has a limited cerebral vascular supply following ligation of the carotid arteries (12). MRI has previously been used to determine 

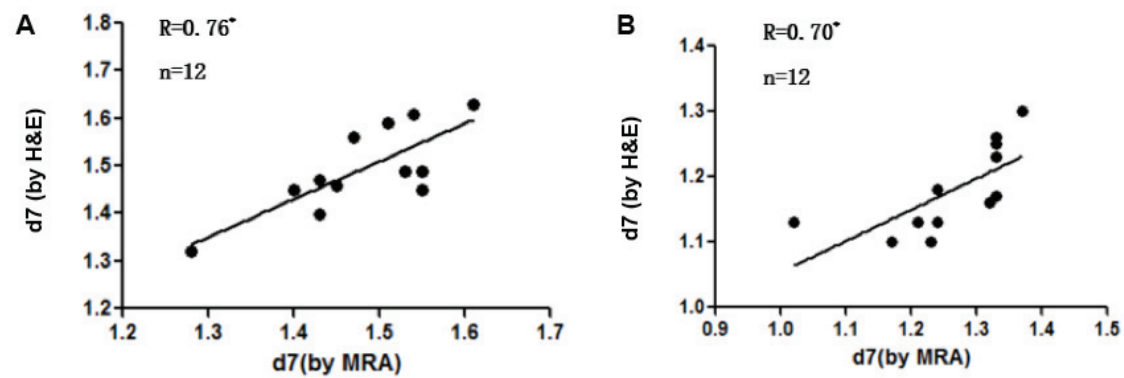

Figure 6. The correlation between H\&E and MRA measuring BA. There was a positive correlation between H\&E and MRA on d7 in the (A) sham group and in the (B) SAH group. ${ }^{*} \mathrm{P}<0.05$. H\&E, hematoxylin and eosin; MRA, magnetic resonance angiography; $\mathrm{d} 7$, day 7.
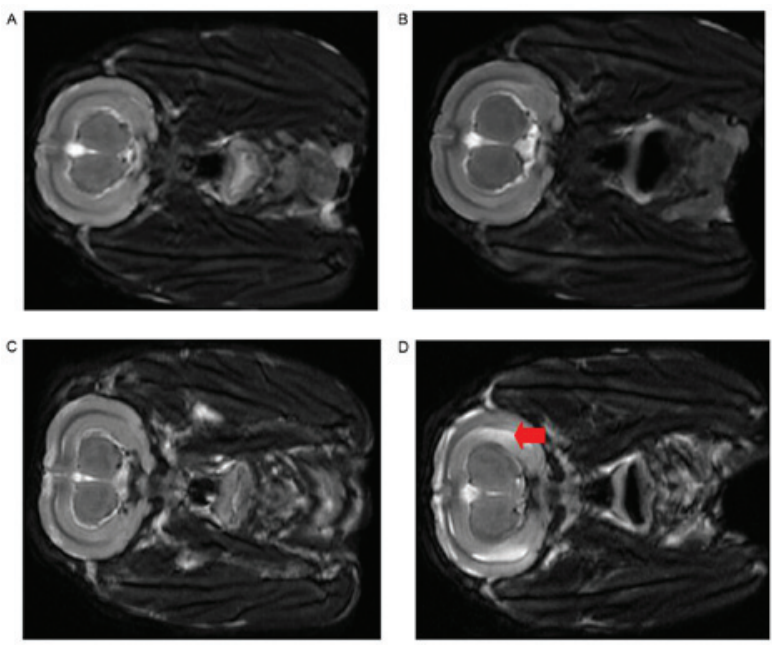

Figure 7. Representative T2-weighted brain MRI images. MRI images of the Sham group on days (A) 0 and (B) 7. MRI images of the subarachnoid hemorrhage group on days (C) 0 and (D) 7. The arrow in Panel D indicates increased MRI signal intensity consistent with ischemic changes. MRI, magnetic resonance imaging.
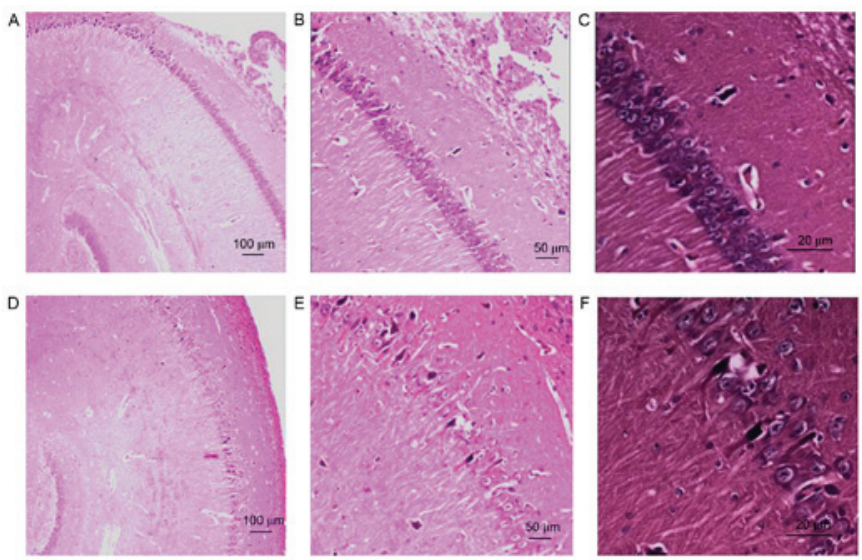

Figure 8. Hematoxylin and eosin staining of the hippocampus. Representative tissues from the Sham group at day 7, demonstrating normal cells and cellular arrangements at magnifications of (A) x40, (B) x100 and (C) x400. Representative tissues from the subarachnoid hemorrhage group at day 7 at magnifications of (D) x40, (E) x100 and (F) x400 revealed karyopyknosis, cytoplasmic staining and smaller cell bodies, consistent with hippocampal ischemia.

the occurrence of cerebral ischemia in a dog CVS model; however, this model was unable to accurately replicate SCVS (29).
Table III. Incidence of hippocampal ischemia assessed by MRA and H\&E.

\begin{tabular}{lcc}
\hline Group & $\begin{array}{c}\text { Hippocampus ischemia Hippocampus ischemia } \\
\text { identified by MRA }\end{array}$ & $\begin{array}{c}\text { identified by H\&E } \\
\text { Sham }\end{array}$ \\
$\begin{array}{l}\text { Subarachnoid } \\
\text { hemorrhage }\end{array}$ & $0 / 12$ & $0 / 12$ \\
& $10 / 12$ & $9 / 12$ \\
\hline
\end{tabular}

MRA, magnetic resonance angiography; H\&E, hematoxylin and eosin.

Endo et al (23) established that SAH causes secondary symptomatic CVS in a rabbit model using New Zealand rabbits. A bilateral carotid artery ligation was performed to block the anterior circulation, followed by injection of blood into the brain. However, neurological function was evaluated based on neurological scores and the subjectivity of this score may have affected the results (23). In their initial study, fresh non-heparinized autologous arterial blood was injected into the cisterna magna twice, 2 weeks following bilateral carotid artery ligation and the degree of cerebral vascular spasm was assessed by digital subtraction angiography (DSA) (23). The femoral artery was ligated for the angiographic procedure, resulting in insufficient blood supply to the lower limbs, which may have affected neurological scores.

In the present study, rabbits were evaluated following bilateral carotid artery ligation using neurological scores and MRA to assess the extent of brain infarction. In a pilot study, the Japanese white rabbit was used to study the same model and it was identified that the mortality rate of rabbits was very high when autologous blood was used (data not shown). Based on this, a rabbit model of SCVS with an improved method for calculating the volume of injected blood was used in the present study. If MRA revealed cerebral ischemia following carotid artery ligation after 2 weeks, the rabbits were excluded from the study. Therefore, MRA was used as a criterion for excluding rabbits with cerebral ischemia. The twice blood injection model typically involves making a subocciptal incision to expose the craniospinal junction; however, the skin and muscle are cut using this method, which increases susceptibility to infection (12). In the present study, apparatus that induced suction was used to establish the model without exposing the craniospinal junction. 
DSA is the gold standard for determining CVS severity and the effectiveness of treatment in humans (30). However, there are several problems with the application of this imaging technique in the rabbit model (14). DSA leads to significant damage due to femoral artery ligation and does not allow dynamic observation of CVS in the BA (31). In previous studies, CTA demonstrated CVS with measurements of BA diameters (32). However, CTA requires injection of a contrast agent that may result in renal dysfunction, a complication that has been widely verified in clinical practice, in addition, it can be difficult to gain venous access multiple times (33). MRA has previously been applied to clinical and animal experiments and may be performed without the need for contrast injection, as demonstrated in the present study (21).

The 3D-TOF MRA sequence allows for dynamic observation of the BA to evaluate arterial spasticity as measured by its quantitative imaging system (21). Based on our previous study (15), it was determined that BA vasospasm occurs 7 days following $\mathrm{SAH}$, suggesting that spasticity was most marked in the first 7 days following SAH. Therefore, as a method of evaluation, MRA is helpful for detecting the incidence of vasospasm in rabbits and for effectively assessing the degree of spasticity.

The results of the present study demonstrate that MRA findings are consistent with the pathological changes that occur in the BA, indicating that MRA may be used to accurately measure the BA diameter. MRA is a noninvasive imaging technique often used to determine the extent of brain damage (34) and previous studies have reported that the extent of brain damage following SAH may be accurately evaluated using MRA (35-37). The hippocampus is the region of the brain most sensitive to ischemia and hypoxia (38). The present study demonstrated that the T2 sequence may be used to identify the extent of brain damage by identifying whether there was a significant increase in the cerebral signal 7 days following surgery-results which are consistent with ischemia in the brain tissue.

There were a number of limitations of the present study. Firstly, although H\&E staining and MRA correctly identified the BA diameter and neurological damage following CVS, errors in measuring the BA diameter may occur without correlative DSA. The diameter of the BA measured by MRA was not compared with the BA diameter measured by DSA, as the duration of anesthesia would have been significantly longer with DSA. Similarly, there may be inconsistencies in the determination of neurological function scores due to inter-observer variability.

To the best of our knowledge, the current study is the first to report the establishment of an SCVS model in Japanese white rabbits using MRA to measure spasticity and brain damage, and to compare the results of MRA with those from pathological examinations. The results of the present study indicate that MRA may be an effective method of evaluating BA vasospasm and hippocampus ischemic change in a rabbit model of SCVS.

\section{Acknowledgements}

The present study was supported by the National Foundation of Natural Science of China (grant nos. 81603685, 81273923 and 81573742) and the Wenzhou Science and Technology Project (grant no. Y20150229).

\section{References}

1. van Gijn J, Kerr RS and Rinkel GJ: Subarachnoid haemorrhage. Lancet 369: 306-318, 2007.

2. Connolly ES Jr, Rabinstein AA, Carhuapoma JR, Derdeyn CP, Dion J, Higashida RT, Hoh BL, Kirkness CJ, Naidech AM, Ogilvy CS, et al: Guidelines for the management of aneurysmal subarachnoid hemorrhage: A guideline for healthcare professionals from the American Heart Association/american Stroke Association. Stroke 43: 1711-1737, 2012.

3. Przybycien-Szymanska MM and Ashley WW Jr: Biomarker discovery in cerebral vasospasm after aneurysmal subarachnoid hemorrhage. J Stroke Cerebrovasc Dis 24: 1453-1464, 2015

4. Steiner T, Juvela S, Unterberg A, Jung C, Forsting M and Rinkel G; European Stroke Organization: European stroke organization guidelines for the management of intracranial aneurysms and subarachnoid haemorrhage. Cerebrovasc Dis 35: 93-112, 2013.

5. Sen J, Belli A, Albon H, Morgan L, Petzold A and Kitchen N: Triple-H therapy in the management of aneurysmal subarachnoid haemorrhage. Lancet Neurol 2: 614-621, 2003.

6. Choudhari K: Prophylactic hyperdynamic postoperative fluid therapy after aneurysmal subarachnoid hemorrhage: A clinical, prospective, randomized, controlled study. Neurosurgery 50: 1170-1172, 2002.

7. Muroi C, Seule M, Mishima K and Keller E: Novel treatments for vasospasm after subarachnoid hemorrhage. Curr Opin Crit Care 18: 119-126, 2012

8. Shimoda M, Takeuchi M, Tominaga J, Oda S, Kumasaka A and Tsugane R: Asymptomatic versus symptomatic infarcts from vasospasm in patients with subarachnoid hemorrhage: Serial magnetic resonance imaging. Neurosurgery 49: 1341-1350, 2001.

9. Udoetuk JD, Stiefel MF, Hurst RW, Weigele JB and LeRoux PD: Admission angiographic cerebral circulation time may predict subsequent angiographic vasospasm after aneurysmal subarachnoid hemorrhage. Neurosurgery 61: 1152-1161, 2007.

10. Lougheed WM and Tom M: A method of introducing blood into the subarachnoid space in the region of the circle of Willis in dogs. Can J Surg 4: 329-337, 1961.

11. Prunell GF, Mathiesen $\mathrm{T}$ and Svendgaard NA: A new experimental model in rats for study of the pathophysiology of subarachnoid hemorrhage. Neuroreport 13: 2553-2556, 2002.

12. Chen S, Klebe KD, Vakhmyanin A, Fujii M and Zhang JH: SAH models: Review, new modification, and prospective. Springer New York, NY, pp255-267, 2014.

13. Otsuji T, Endo S, Hirashima Y, Nishijima M and Takaku A: An experimental model of symptomatic vasospasm induced by oxyhemoglobin in rabbits. Stroke 25: 657-662, 1994.

14. Titova E, Ostrowski RP, Zhang JH and Tang J: Experimental models of subarachnoid hemorrhage for studies of cerebral vasospasm. Neurol Res 31: 568-581, 2009.

15. Yunchang M, Qinxue D, Binbin J, Xin H, Lili Y, Linbi C, Wujun G, Pengbo Z and Junlu W: Human tissue kallikrein ameliorates cerebral vasospasm in a rabbit model of subarachnoid hemorrhage. Neurol Res 37: 1082-1095, 2015.

16. Stacul F, van der Molen AJ, Reimer P, Webb JA, Thomsen HS, Morcos SK, Almén T, Aspelin P, Bellin MF, Clement O, et al: Contrast induced nephropathy: Updated ESUR contrast media safety committee guidelines. Eur Radiol 21: 2527-2541, 2011.

17. Seeliger E, Sendeski M, Rihal CS and Persson PB: Contrast-induced kidney injury: Mechanisms, risk factors, and prevention. Eur Heart J 33: 2007-2015, 2012.

18. Kruk M: Measuring behaviour into the twenty-first century. Trends Neurosci 20: 187-189, 1997.

19. Taufique Z, May T, Meyers E, Falo C, Mayer SA, Agarwal S, Park S, Connolly ES, Claassen J and Schmidt JM: Predictors of poor quality of life 1 year after subarachnoid hemorrhage. Neurosurgery 78: 256-264, 2016.

20. Yang JP, Liu HJ and Liu RC: A modified rabbit model of stroke: Evaluation using clinical MRI scanner. Neurol Res 31: 1092-1096, 2009.

21. Majoie CB, Sprengers ME, van Rooij WJ, Lavini C, Sluzewski M, van Rijn JC and den Heeten GJ: MR angiography at 3T versus digital subtraction angiography in the follow-up of intracranial aneurysms treated with detachable coils. AJNR Am J Neuroradiol 26: 1349-1356, 2005.

22. van Amerongen MJ, Boogaarts HD, de Vries J, Verbeek AL, Meijer FJ, Prokop M and Bartels RH: MRA versus DSA for follow-up of coiled intracranial aneurysms: A meta-analysis. AJNR Am J Neuroradiol 35: 1655-1661, 2014. 
23. Endo S, Branson PJ and Alksne JF: Experimental model of symptomatic vasospasm in rabbits. Stroke 19: 1420-1425, 1988.

24. Kertmen H, Gürer B, Yilmaz ER, Arikok AT, Kanat MA, Ergüder BI and Sekerci Z: The comparative effects of recombinant human erythropoietin and darbepoetin-alpha on cerebral vasospasm following experimental subarachnoid hemorrhage in the rabbit. Acta Neurochir (Wien) 156: 951-962, 2014.

25. Bederson JB, Connolly ES Jr, Batjer HH, Dacey RG, Dion JE, Diringer MN, Duldner JE Jr, Harbaugh RE, Patel AB and Rosenwasser RH; American Heart Association: Guidelines for the management of aneurysmal subarachnoid hemorrhage: A statement for healthcare professionals from a special writing group of the Stroke Council, American Heart Association. Stroke 40: 994-1025, 2009.

26. Venti M: Subarachnoid and intraventricular hemorrhage. Front Neurol Neurosci 30: 149-153, 2012.

27. Harrod CG, Bendok BR and Batjer HH: Prediction of cerebral vasospasm in patients presenting with aneurysmal subarachnoid hemorrhage: A review. Neurosurgery 56: 633-654, 2005.

28. Crowley RW, Medel R, Dumont AS, Ilodigwe D, Kassell NF, Mayer SA, Ruefenacht D, Schmiedek P, Weidauer S, Pasqualin A and Macdonald RL: Angiographic vasospasm is strongly correlated with cerebral infarction after subarachnoid hemorrhage. Stroke 42: 919-923, 2011.

29. Jadhav V, Sugawara T, Zhang J, Jacobson P and Obenaus A Magnetic resonance imaging detects and predicts early brain injury after subarachnoid hemorrhage in a canine experimental model. J Neurotrauma 25: 1099-1106, 2008.

30. Kumar A, Kato Y, Hayakawa M, Junpei O, Watabe T, Imizu S, Oguri D and Hirose Y: Recent advances in diagnostic approaches for sub-arachnoid hemorrhage. Asian J Neurosurg 6: 94-98, 2011.

31. Pryor JC, Setton A, Nelson PK and Berenstein A: Complication of diagnostic cerebral angiography and tips on avoidance. Neuroimaging Clin N Am 6: 751-758, 1996.
32. Laslo AM, Eastwood JD, Chen FX and Lee TY: Dynamic CT perfusion imaging in subarachnoid hemorrhage-related vasospasm. AJNR Am J Neuroradiol 27: 624-631, 2006.

33. Hotta K, Sorimachi T, Osada T, Baba T, Inoue G, Atsumi H, Ishizaka $\mathrm{H}$, Matsuda $\mathrm{M}$, Hayashi $\mathrm{N}$ and Matsumae $\mathrm{M}$ : Risks and benefits of CT angiography in spontaneous intracerebral hemorrhage. Acta Neurochir (Wien) 156: 911-917, 2014.

34. Pierot L, Portefaix C, Rodriguez-Régent C, Gallas S, Meder JF and Oppenheim C: Role of MRA in the detection of intracranial aneurysm in the acute phase of subarachnoid hemorrhage. J Neuroradiol 40: 204-210, 2013.

35. Frontera JA, Ahmed W, Zach V, Jovine M, Tanenbaum L, Sehba F, Patel A, Bederson JB and Gordon E: Acute ischaemia after subarachnoid haemorrhage, relationship with early brain injury and impact on outcome: A prospective quantitative MRI study. J Neurol Neurosurg Psychiatry 86: 71-78, 2015.

36. Griffiths PD, Wilkinson ID, Mitchell P, Patel MC, Paley MN, Romanowski CA, Powell T, Hodgson TJ, Hoggard N and Jellinek D: Multimodality MR imaging depiction of hemodynamic changes and cerebral ischemia in subarachnoid hemorrhage. AJNR Am J Neuroradiol 22: 1690-1677, 2001.

37. Wani AA, Phadke R, Behari S, Sahu R, Jaiswal A and Jain V: Role of diffusion-weighted MRG in predicting outcome in subarachnoid hemorrhage due to anterior communicating artery aneurysms. Turk Neurosurg 18: 10-16, 2008.

38. Huang CC, Lai CJ, Tsai MH, Wu YC, Chen KT, Jou MJ, Fu PI, Wu CH and Wei IH: Effects of melatonin on the nitric oxide system and protein nitration in the hypobaric hypoxic rat hippocampus. BMC Neurosci 16: 61, 2015.

(c) (i) $(9$ This work is licensed under a Creative Commons

C. Attribution-NonCommercial-NoDerivatives 4.0 International (CC BY-NC-ND 4.0) License. 